\title{
On time-domain methods for milling stability analysis
}

\author{
DING Han ${ }^{1 *}$, DING Ye ${ }^{2} \&$ ZHU LiMin ${ }^{2}$ \\ ${ }^{1}$ State Key Laboratory of Digital Manufacturing Equipment and Technology, Huazhong University of Science and Technology, Wuhan 430074, \\ China; \\ ${ }^{2}$ State Key Laboratory of Mechanical System and Vibration, Shanghai Jiao Tong University, Shanghai 200240, China
}

Received June 25, 2012; accepted September 12, 2012

\begin{abstract}
As a basic and advanced machining technique, the high-speed milling process plays an important role in realizing the goal of high performance manufacturing. From the viewpoint of machining dynamics, obtaining chatter-free machining parameters is a prerequisite to guaranteeing machining accuracy and improving machining efficiency. This paper gives an overview on recent progress in time domain semi-analytical methods for chatter stability analysis of milling processes. The state of art methods of milling stability prediction in milling processes and their applications are introduced in detail. The bottlenecks involved are analyzed, and potential solutions are discussed. Finally, a brief prospect on future works is presented.
\end{abstract}

milling dynamics, stability analysis, semi-analytical method

Citation: $\quad$ Ding H, Ding Y, Zhu L M. On time-domain methods for milling stability analysis. Chin Sci Bull, 2012, 57: 4336-4345, doi: 10.1007/s11434-012-5499-y

High speed milling is widely utilized in manufacturing of complex surfaces for the fields of aerospace, ship, automotive, dies and molds etc., due to its advantage of obtaining high machining accuracy and high material removal rate with keeping low-amplitude cutting forces. Regarding to modeling of machining processes, the research topics on high speed milling can be categorized as: tool path planning, machining dynamics and machining physical modeling. Tool path planning is to plan the tool path according to the workpiece model, machining strategy and required machined error [1-5]. From the viewpoint of machining dynamics, the relative vibration between the workpiece and cutter in the milling process is the main reason of reducing product surface quality and limiting the production efficiency. To suppress the vibration impact by optimizing the machining parameters, the following two questions need to be addressed.

(1) Stability analysis based on the dynamics of milling processes. As far as chatter vibrations are concerned, there are four different mechanisms: regeneration [6], mode coupling [7], friction [8] and thermo-mechanics of chip for-

*Corresponding author (email: dinghan@mail.hust.edu.cn) mation [9]. In milling processes, regenerative chatter is the most common form of self-excited vibration to reduce surface quality and machining efficiency [10,11]. The works on analysis of the stability of milling processes focus on calculating the stability boundary of the machining parameters based on the dynamic models characterizing the milling processes.

(2) Machining accuracy analysis on the basis of stability analysis. Due to forced vibrations in milling processes, chatter-free machining parameters cannot ensure high performance machining. Vibration-induced surface errors can be classified as surface location error (SLE) [12] and surface roughness [13]. It is essential to take the stability and dynamical errors into account in machining optimization models to achieve high performance milling.

\section{Milling dynamics model}

Without loss of generality, two degree-of-freedom (DOF) lumped parameter dynamics model of the cutter is shown in Figure 1 . In this figure, $\phi_{j}(t)$ is the angular position of the $j$ th tooth, $\Omega$ is the spindle speed $\left(\mathrm{r} \mathrm{min}^{-1}\right), a_{e}$ is the radial depth 


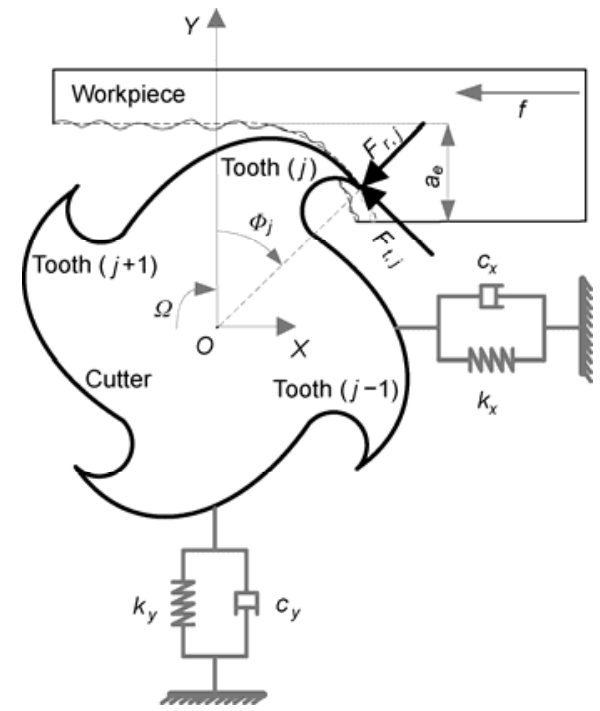

Figure 1 Two DOF lumped parameter dynamics model of the milling process.

of cut, $f$ is the feed direction of the workpiece with respect to the cutter, $F_{t, j}$ and $F_{r, j}$ are the tangential and normal cutting force components for the $j$ th tooth, respectively.

The governing equation of the milling process shown in Figure 1 can be formulated as the following delayed differential equation (DDE) with the periodic coefficient [14-16]:

$$
\boldsymbol{M} \ddot{\boldsymbol{q}}(t)+\boldsymbol{C} \dot{\boldsymbol{q}}(t)+\boldsymbol{K} \boldsymbol{q}(t)=\boldsymbol{K}_{c}(t)[\boldsymbol{q}(t)-\boldsymbol{q}(t-T)],
$$

where $\boldsymbol{M}, \boldsymbol{C}, \boldsymbol{K}$ and $\boldsymbol{q}(t)$ represent the modal matrix, damping matrix, stiffness matrix and modal vector of the cutter, respectively. $T$ is the time delay, equal to tooth passing period, i.e., $T=60 /(N \Omega)$, where $N$ is the number of the cutter teeth. $\boldsymbol{K}_{c}(t)$ is the cutting force coefficient matrix (referring to [14]), and $\boldsymbol{K}_{c}(t)=\boldsymbol{K}_{c}(t+T)$, defined as

$$
\boldsymbol{K}_{c}(t)=\left[\begin{array}{ll}
-a_{p} h_{x x}(t) & -a_{p} h_{x y}(t) \\
-a_{p} h_{y x}(t) & -a_{p} h_{y y}(t)
\end{array}\right]
$$

where $a_{p}$ is the axial depth of cut, $h_{x x}(t), h_{x y}(t), h_{y x}(t)$ and $h_{y y}(t)$ are

$$
\begin{aligned}
h_{x x}(t)= & \sum_{j=1}^{N} g\left(\phi_{j}(t)\right) \sin \left(\phi_{j}(t)\right)\left[K_{t} \cos \left(\phi_{j}(t)\right)\right. \\
& \left.+K_{n} \sin \left(\phi_{j}(t)\right)\right], \\
h_{x y}(t)= & \sum_{j=1}^{N} g\left(\phi_{j}(t)\right) \cos \left(\phi_{j}(t)\right)\left[K_{t} \cos \left(\phi_{j}(t)\right)\right. \\
& \left.+K_{n} \sin \left(\phi_{j}(t)\right)\right], \\
h_{y x}(t)= & \sum_{j=1}^{N} g\left(\phi_{j}(t)\right) \sin \left(\phi_{j}(t)\right)\left[-K_{t} \sin \left(\phi_{j}(t)\right)\right. \\
& \left.+K_{n} \cos \left(\phi_{j}(t)\right)\right],
\end{aligned}
$$

$$
\begin{aligned}
h_{y y}(t)= & \sum_{j=1}^{N} g\left(\phi_{j}(t)\right) \cos \left(\phi_{j}(t)\right)\left[-K_{t} \sin \left(\phi_{j}(t)\right)\right. \\
& \left.+K_{n} \cos \left(\phi_{j}(t)\right)\right] .
\end{aligned}
$$

In eqs. (3)-(6), $K_{t}$ and $K_{n}$ are the tangential and normal linearized cutting force coefficients, respectively. $\phi_{j}(t)$ is defined by

$$
\phi_{j}(t)=(2 \pi \Omega / 60) t+(j-1) \cdot 2 \pi / N .
$$

The window function $g\left(\phi_{j}(t)\right)$ is defined as

$$
g\left(\phi_{j}(t)\right)= \begin{cases}1 & \text { if } \phi_{\mathrm{st}}<\phi_{j}(t) \bmod 2 \pi<\phi_{\mathrm{ex}} \\ 0 & \text { otherwise }\end{cases}
$$

where $\phi_{\mathrm{st}}$ and $\phi_{\mathrm{ex}}$ are the start and exit angles of the $j$ th tooth, respectively. For down-milling, $\phi_{\mathrm{st}}=\arccos \left(2 a_{e} / D\right.$ $-1)$, and $\phi_{\mathrm{ex}}=\pi$; for up-milling, $\phi_{\mathrm{st}}=0$ and $\phi_{\mathrm{ex}}=$ $\arccos \left(1-2 a_{e} / D\right)$, where $a_{e} / D$ is the radial immersion ratio (the radial depth of cut /the cutter diameter).

Denoting by $\boldsymbol{p}(t)=\boldsymbol{M} \dot{\boldsymbol{q}}(t)+\boldsymbol{C} \boldsymbol{q}(t) / 2$ and $\boldsymbol{x}(t)=\left[\begin{array}{c}\boldsymbol{q}(t) \\ \boldsymbol{p}(t)\end{array}\right]$, eq. (1) can be rewritten as the state-space form

$$
\dot{\boldsymbol{x}}(t)=\boldsymbol{A x}(t)+\boldsymbol{B}(t)[\boldsymbol{x}(t)-\boldsymbol{x}(t-T)],
$$

where

$$
\boldsymbol{A}=\left[\begin{array}{cc}
-\boldsymbol{M}^{-1} \boldsymbol{C} / 2 & \boldsymbol{M}^{-1} \\
\boldsymbol{C} \boldsymbol{M}^{-1} \boldsymbol{C} / 4-\boldsymbol{K} & -\boldsymbol{C M}^{-1} / 2
\end{array}\right], \boldsymbol{B}(t)=\left[\begin{array}{cc}
\boldsymbol{0} & \boldsymbol{0} \\
\boldsymbol{K}_{c}(t) & \boldsymbol{0}
\end{array}\right] .
$$

Due to the complexity of the geometry of cutters and interface dynamics of cutter-holder-spindle, it is challenging to obtain the modal parameters $\boldsymbol{M}, \boldsymbol{C}$ and $\boldsymbol{K}$ by using analytical or numerical methods. Nowadays, there are two methods for obtaining the frequency response functions (FRFs) of the cuter as follows. (1) Experimental modal testing method [14]. With the advantage of being easy to implement, the main disadvantage of this method is that the modal testing experiment should be conducted for each combination of cutter-holder-spindle. (2) Experimentalanalytical method. Schmitz et al. $[17,18]$ proposed the receptance coupling substructure analysis (RCSA) method. By using this method, the interface parameters of cutterholder-spindle can be determined and the FRFs can be analytically calculated. Hence, the work burden of modal testing experiments can be reduced dramatically.

To determine the cutting force parameters $K_{t}$ and $K_{n}$, the main methods can be categorized as follows. (1) The mechanistic based method [14,19]. For the chosen cutter and workpiece, the mechanistic model can be calibrated by using measured cutting forces from several cutting tests. (2) The orthogonal cutting data based method [20,21]. The experimental times could be reduced, since the cutting force 
coefficients can be calculated based on the orthogonal cutting database.

As for the multi-mode effect of the cutter and the workpiece in the milling processes, refer to [16] and [22], respectively. As for the mode-coupling effect, refer to [23].

It is worth noting that the stability of milling processes can also be directly determined via analysis of process signals, e.g., the cutting force signals [24] or the motor current signals [25], acquired from experiments. The advantage of this method is that no dynamic model is required. However, the disadvantage is also obvious, i.e., a large number of experiments due to different machining parameters should be conducted to determine the stability boundary. The main scope of this paper is to review the methods for stability analysis and machining accuracy analysis based on eq. (9).

\section{Model based stability analysis and machining error calculation}

Compared with traditional periodic systems without time delay, the monodromy operator and phase space of eq. (9) are both of infinite dimensions, which results in the essential difficulty for stability analysis of this system. Many kinds of approximate methods have been proposed for solving this problem. Generally, they can be classified as two kinds: numerical methods and analytical (or semianalytical) methods.

\subsection{Stability analysis methods}

(1) Numerical methods. The key idea of the numerical methods is to calculate the time-domain response of the DDE, then distinguish instable vibrations according to some stability criteria.

Sridhar et al. [26] proposed a numerical algorithm for the general milling process. Tlusty et al. [27,28] proposed a finite difference based simulation procedure for chatter stability analysis in milling. Smith and Tlusty [29] suggested the Peak-to-Peak (PTP) diagrams for time-domain milling stability analysis. Davies et al. [30,31] proposed a discrete map based method for stability prediction in low radial immersion milling. Campomanes and Altintas [32] proposed an improved time domain simulation method and the instability criterion by defining the ratio of the maximum dynamic uncut chip thickness to the maximum static uncut chip thickness. Li and Liu [33] presented a time domain simulation method for milling processes based on the Runge-Kutta algorithm.

The numerical methods can be used for the complex cases considering nonlinear cutting force models and timevarying radial immersion boundaries etc. The main disadvantage of this kind of methods is that the computational efficiency is generally much lower than analytical (or semianalytical) methods [10].
(2) Analytical (semi-analytical) methods. The essential idea of analytical (or semi-analytical) methods is to approximate the original DDE, yielding finite monodromy operators to approximate the original infinite one. The main advantage of this kind of methods is to efficiently predict the chatter stability with acceptable approximate errors.

Minis and Yanushevsky [34] used the Nyquist criterion to analyze the milling stability based on the theory of periodic differential equations. Altintas and Budak [35,36] proposed the zeroth order approximation (ZOA) method. The analytical solution of the critical axial depth of cut for one fixed spindle speed is obtained by this method [35]. The main advantage of this method is its computational efficiency. However, it cannot predict the flip bifurcation in low radial immersion milling. Merdol and Altintas [37] then proposed the multi frequency solution to solve this problem. Since the iteration procedure for chatter frequencies is employed, there is no analytical solution. In these years, the team of Professor Altintas has applied the frequency domain method in the cases of ball-end milling, milling with variable pitch cutters, plunge milling, etc.

Bayly et al. [38] proposed the temporal finite element analysis (TFEA) method for single DOF milling model, then generalized it to two DOF case. This method assumes the form of the solution on the time elements, and then constructs the Floquet transition matrix based on the weighted residual method. Mann and Patel [39] presented the TFEA method in the state space form. Garg et al. [40] generalized the original TFEA for stability analysis of general time-delayed systems with parametric excitation, employing multi-grid techniques.

Insperger and Stépán [41,42] proposed the zeroth order semi-discretization method (SDM) for milling stability analysis. By using the zeroth approximation of the time delay item and the time-periodic item on one time interval, this method simplifies the original DDE as a series of ordinary differential equations (ODEs). On this basis, the approximate Floquet transition matrix is constructed. To improve the calculation accuracy, Insperger and Stépán then proposed the first order SDM [43]. In this procedure, the time delayed item is approximated by the linear polynomial. Long et al. utilized the SDM to analyze the milling stability considering the loss-of-contact effects between the workpiece and the tool [44] and the varying spindle speed effect [45]. Li et al. [46] proposed an improved SDM by using the Magnus-Gaussian series. Song et al. used the SDM to calculate the chatter stability considering the runout effect of the tool [47] and the feed effect on stability [48]. Wan et al. [49] proposed the unified SDM taking the multi-time delay effect into account.

Besides the aforementioned methods, Olgac and Hosek [50] proposed the root locus plot analysis method for prediction of chatter stability, and then generalized to variable pitch milling [51]. Ulsoy et al. [52,53] proposed the Lambert $W$ function based method for machining stability anal- 
ysis. Butcher et al. presented the Chebyshev polynomial based method [54] and Chebyshev collocation method [55] for milling stability prediction, respectively.

\subsection{Machining error calculation methods}

For calculation of surface location error, there exist the numerical method [12], the frequency domain method [56], the TFEA method [16,57,58] and the harmonic balance method [59]. It is worth noting that the TFEA method can be used for simultaneous prediction of milling stability and SLE [58]. It is much efficient than other methods, since the fixed point technique is employed to calculate the SLE.

There are some shortcomings of these widely utilized stability analysis methods in computational efficiency or generality, respectively. Since high speed milling technique has the characteristics of high speed, time-varying feed speed and radial immersion, the high-efficient and general calculation method is urgently demanded for simultaneous prediction of chatter stability and SLE.

\section{Time-domain semi-analytical methods}

The key idea of time-domain semi-analytical methods is to approximate the original DDE by using some reasonable simplifications, and then construct the corresponding Floquet transition matrices according to the simplified forms. At last, the stability of the system can be evaluated by using the Floquet theory. From the viewpoint of modern numerical analysis, there exist three kinds of numerical techniques to calculate the dynamic response of dynamical systems, i.e., the differential equation based methods, the variational method based methods and the integral equation based methods. The SDM and TFEA correspond to the first two kinds, respectively. Inspired by the numerical methods for integral equations with high precision, we proposed the fulldiscretization method (FDM) [61] and numerical integration method [62] in the framework of integral equations.

\subsection{Differential equation based methods}

In the SDM [42], the first step to analyze eq. (9) is to discretize the time period $T$ as $m$ small time intervals, i.e., $T=m \tau$, where $m$ is one of the positive integers. On each interval $k \tau \leqslant t \leqslant(k+1) \tau, \quad(k=0, \ldots, m)$, with the initial condition $\boldsymbol{x}_{k}=\boldsymbol{x}(k \tau)$, by discretizing the time delayed item $\boldsymbol{x}(t-T)$, performing zeroth order average of the delayed item and the periodic matrix $\boldsymbol{B}(t)$ on each time interval, respectively, eq. (9) can be simplified as the following ODE,

$$
\dot{\boldsymbol{x}}_{k}(t)=\left(\boldsymbol{A}+\boldsymbol{B}_{k}\right) \boldsymbol{x}_{k}(t)-\frac{1}{2} \boldsymbol{B}_{k}\left[\boldsymbol{x}_{k-m+1}+\boldsymbol{x}_{k-m}\right],
$$

where

$$
\boldsymbol{B}_{k}=\frac{1}{\tau} \int_{t_{k}}^{t_{k+1}} \boldsymbol{B}(t) \mathrm{d} t
$$

From eq. (10), $\boldsymbol{x}_{k+1}=\boldsymbol{x}(k \tau+\tau)$ can be expressed as

$$
\boldsymbol{x}_{k+1}=\mathrm{e}^{\left(\boldsymbol{A}+\boldsymbol{B}_{k}\right) \tau} \boldsymbol{x}_{k}-\frac{1}{2}\left[\mathrm{e}^{\left(\boldsymbol{A}+\boldsymbol{B}_{k}\right) \tau}-\boldsymbol{I}\right]\left[\boldsymbol{A}+\boldsymbol{B}_{k}\right]^{-1} \boldsymbol{B}_{k}\left[\boldsymbol{x}_{k-m+1}+\boldsymbol{x}_{k-m}\right] .
$$

Eq. (12) can be rewritten as

$$
\boldsymbol{y}_{k+1}=\boldsymbol{S}_{k} \boldsymbol{y}_{k},
$$

where $\boldsymbol{y}_{k}=\operatorname{col}\left(\boldsymbol{x}_{k}, \boldsymbol{x}_{k-1}, \ldots, \boldsymbol{x}_{k-m}\right)$,

$$
\boldsymbol{S}_{k}=\left[\begin{array}{cccccc}
\mathrm{e}^{\left(A+\boldsymbol{B}_{k}\right) \tau} & \boldsymbol{0} & \cdots & \boldsymbol{0} & -\frac{1}{2}\left[\mathrm{e}^{\left(\boldsymbol{A}+\boldsymbol{B}_{k}\right) \tau}-\boldsymbol{I}\right]\left[\boldsymbol{A}+\boldsymbol{B}_{k}\right]^{-1} \boldsymbol{B}_{k} & -\frac{1}{2}\left[\mathrm{e}^{\left(\Lambda+\boldsymbol{B}_{k}\right) \tau}-\boldsymbol{I}\right]\left[\boldsymbol{A}+\boldsymbol{B}_{k}\right]^{-1} \boldsymbol{B}_{k} \\
\boldsymbol{I} & \boldsymbol{0} & \cdots & \boldsymbol{0} & \boldsymbol{0} & \boldsymbol{0} \\
\boldsymbol{0} & \boldsymbol{I} & \cdots & \boldsymbol{0} & \boldsymbol{0} & \boldsymbol{0} \\
\vdots & \vdots & \ddots & \vdots & \vdots & \vdots \\
\boldsymbol{0} & \boldsymbol{0} & \cdots & \boldsymbol{I} & \boldsymbol{0} & \boldsymbol{0} \\
\boldsymbol{0} & \boldsymbol{0} & \cdots & \boldsymbol{0} & \boldsymbol{I} & \boldsymbol{0}
\end{array}\right] .
$$

On this basis, the Floquet matrix can be constructed by using eq. (13), i.e.,

$$
\boldsymbol{\Psi}_{1}=\boldsymbol{S}_{m-1} \boldsymbol{S}_{m-2} \cdots \boldsymbol{S}_{0} .
$$

According to eq. (14), the chatter stability can be predicted based on the Floquet theory, i.e.,

$$
\max \left(\left|\Lambda\left(\boldsymbol{\Psi}_{1}\right)\right|\right) \begin{cases}<1 & \text { stable } \\ =1 & \text { stable boundary } \\ >1 & \text { instable }\end{cases}
$$

where $\Lambda(\cdot)$ denotes the spectral radius of a matrix.

\subsection{Weighted residual method based methods}

In the TFEA method [63], denote by $t_{0}$ the time cutter leaves the workpiece, $t_{f}$ the duration of the free vibration after the cutter leaves the workpiece, $t_{c}$ the duration of the forced vibration in cut. Firstly, the duration of forced vibration $\left[t_{0}+t_{f}, t_{0}+T\right]$ is equally divided into $m$ parts, where $\tau=\left(T-t_{f}\right) / m$. Denote by the sampling points by $t_{i}=t_{0}+t_{f}+(i-1) \tau$, where $i=1, \cdots, m+1$ and $t_{1}=t_{0}+t_{f}$.

On the interval $\left[t_{i}, t_{i+1}\right], i=1, \cdots, m$, the assumed state function and time-delayed function are

$$
\begin{array}{r}
\boldsymbol{x}_{i}(t)=\sum_{k=1}^{3} \boldsymbol{a}_{i k}^{n} \varsigma_{k}(\sigma), \\
\boldsymbol{x}_{i}(t-T)=\sum_{k=1}^{3} \boldsymbol{a}_{i k}^{n-1} \varsigma_{k}(\sigma),
\end{array}
$$

where $\varsigma_{k}(\sigma)$ is the Legendre polydomial, $\sigma$ is the local time $0 \leqslant \sigma \leqslant \tau, \quad \boldsymbol{a}_{i k}^{n}$ represents the $k$ th state on the interval $\left[t_{i}, t_{i+1}\right]$ in the $n$th period. 
Without loss of generality, let $m=2$, and substitute eqs. (16), (17) into eq. (9), the following algebraic equation can be derived based on the weighed residual method:

$$
\begin{aligned}
& {\left[\begin{array}{ccccc}
\boldsymbol{I} & \boldsymbol{0} & \boldsymbol{0} & \boldsymbol{0} & \boldsymbol{0} \\
\boldsymbol{N}_{11}^{1} & \boldsymbol{N}_{12}^{1} & \boldsymbol{N}_{13}^{1} & \boldsymbol{0} & \boldsymbol{0} \\
\boldsymbol{N}_{21}^{1} & \boldsymbol{N}_{22}^{1} & \boldsymbol{N}_{23}^{1} & \boldsymbol{0} & \boldsymbol{0} \\
\boldsymbol{0} & \boldsymbol{0} & \boldsymbol{N}_{11}^{2} & \boldsymbol{N}_{12}^{2} & \boldsymbol{N}_{13}^{2} \\
\boldsymbol{0} & \boldsymbol{0} & \boldsymbol{N}_{21}^{2} & \boldsymbol{N}_{22}^{2} & \boldsymbol{N}_{23}^{2}
\end{array}\right]\left[\begin{array}{l}
\boldsymbol{a}_{11}^{n} \\
\boldsymbol{a}_{12}^{n} \\
\boldsymbol{a}_{21}^{n} \\
\boldsymbol{a}_{22}^{n} \\
\boldsymbol{a}_{23}^{n}
\end{array}\right]} \\
& =\left[\begin{array}{ccccc}
\boldsymbol{0} & \boldsymbol{0} & \boldsymbol{0} & \boldsymbol{0} & \exp \left(\boldsymbol{A} t_{f}\right) \\
\boldsymbol{P}_{11}^{1} & \boldsymbol{P}_{12}^{1} & \boldsymbol{P}_{13}^{1} & \boldsymbol{0} & \boldsymbol{0} \\
\boldsymbol{P}_{21}^{1} & \boldsymbol{P}_{22}^{1} & \boldsymbol{P}_{23}^{1} & \boldsymbol{0} & \boldsymbol{0} \\
\boldsymbol{0} & \boldsymbol{0} & \boldsymbol{P}_{11}^{2} & \boldsymbol{P}_{12}^{2} & \boldsymbol{P}_{13}^{2} \\
\boldsymbol{0} & \boldsymbol{0} & \boldsymbol{P}_{21}^{2} & \boldsymbol{P}_{22}^{2} & \boldsymbol{P}_{23}^{2}
\end{array}\right]\left[\begin{array}{l}
\boldsymbol{a}_{11}^{n-1} \\
\boldsymbol{a}_{12}^{n-1} \\
\boldsymbol{a}_{21}^{n-1} \\
\boldsymbol{a}_{22}^{n-1} \\
\boldsymbol{a}_{23}^{n-1}
\end{array}\right],
\end{aligned}
$$

where

$$
\begin{gathered}
\boldsymbol{N}_{p k}^{i}=\int_{0}^{\tau}\left(\boldsymbol{I} \dot{\boldsymbol{\zeta}}_{k}(\sigma)-(\boldsymbol{A}+\boldsymbol{B}) \boldsymbol{\zeta}_{k}(\sigma)\right) \psi_{p}(\sigma) \mathrm{d} \sigma, \\
\boldsymbol{P}_{p k}^{i}=\int_{0}^{\tau} \boldsymbol{B} \varsigma_{k}(\sigma) \psi_{p}(\sigma) \mathrm{d} \sigma,
\end{gathered}
$$

$\psi_{p}(\sigma)$ is the Legendre polynomial, i.e., $\psi_{1}(\sigma)=1$ and $\psi_{2}(\sigma)=2(\sigma / \tau)-1$.

Eq. (18) can be expressed as the compact form $\boldsymbol{G} \boldsymbol{a}_{n}=\boldsymbol{H} \boldsymbol{a}_{n-1}$. Then the Floquet matrix is

$$
\boldsymbol{\Psi}_{2}=\boldsymbol{G}^{-1} \boldsymbol{H}
$$

In similar, the stability can be predicted by using the spectral radius of $\boldsymbol{\Psi}_{2}$ according to the Floquet theory.

\subsection{Integral equation based methods}

In eq. (9), regarding the item $\boldsymbol{B}(t)[\boldsymbol{x}(t)-\boldsymbol{x}(t-T)]$ as the non-homogenous item of the equation $\dot{\boldsymbol{x}}(t)=\boldsymbol{A} \boldsymbol{x}(t)$, the solution of eq. (9) can be expressed as

$$
\begin{aligned}
\boldsymbol{x}(t)= & \exp \left[\boldsymbol{A}\left(t-t_{0}\right)\right] \boldsymbol{x}\left(t_{0}\right) \\
& +\int_{t_{0}}^{t}\{\exp [\boldsymbol{A}(t-\xi)] \boldsymbol{B}(\xi)[\boldsymbol{x}(\xi)-\boldsymbol{x}(\xi-T)]\} \mathrm{d} \xi
\end{aligned}
$$

where $\boldsymbol{x}\left(t_{0}\right)$ is the state of the system at $t=t_{0}$.

(1) Full-discretization method (FDM). Similar to the SDM, the first step of the FDM [61] for eq. (22) is also equally dividing the period $T$ as $m$ parts, i.e., $T=m \tau$, where $m$ is one of the integral numbers. On each time interval $[k \tau,(k+1) \tau],(k=0, \cdots, m)$, regarding $\boldsymbol{x}_{k}=\boldsymbol{x}(k \tau)$ as the initial condition, eq. (22) can be rewritten as $(0 \leqslant t \leqslant \tau)$

$$
\begin{aligned}
\boldsymbol{x}(k \tau+t)= & \exp (\boldsymbol{A} t) \boldsymbol{x}(k \tau)+\int_{0}^{t} \exp (\boldsymbol{A} \xi) \boldsymbol{B}(k \tau+t-\xi) \\
& \times[\boldsymbol{x}(k \tau+t-\xi)-\boldsymbol{x}(k \tau+t-\xi-T)]\} \mathrm{d} \xi
\end{aligned}
$$

As for eq. (23), letting $t=\tau$, and performing linear interpolations for $\boldsymbol{B}(k \tau+\tau-\xi), \quad \boldsymbol{x}(k \tau+\tau-\xi)$ and $\boldsymbol{x}(k \tau$ $+\tau-\xi-T)$ on the interval $[k \tau,(k+1) \tau], \boldsymbol{x}_{k+1}$ can be derived as

$$
\begin{aligned}
\boldsymbol{x}_{k+1}= & {\left[\boldsymbol{I}-\boldsymbol{F}_{k+1}\right]^{-1}\left(\boldsymbol{F}_{0}+\boldsymbol{F}_{0, k}\right) \boldsymbol{x}_{k} } \\
& +\left[\boldsymbol{I}-\boldsymbol{F}_{k+1}\right]^{-1} \boldsymbol{F}_{m-1} \boldsymbol{x}_{k+1-m}+\left[\boldsymbol{I}-\boldsymbol{F}_{k+1}\right]^{-1} \boldsymbol{F}_{m} \boldsymbol{x}_{k-m},
\end{aligned}
$$

where

$$
\begin{gathered}
\boldsymbol{F}_{0}=\boldsymbol{\Phi}_{0}, \\
\boldsymbol{F}_{0, k}=\left(\boldsymbol{\Phi}_{2} / \tau\right) \boldsymbol{B}_{0}^{(k)}+\left(\boldsymbol{\Phi}_{3} / \tau\right) \boldsymbol{B}_{1}^{(k)}, \\
\boldsymbol{F}_{k+1}=\left(\boldsymbol{\Phi}_{1}-\boldsymbol{\Phi}_{2} / \tau\right) \boldsymbol{B}_{0}^{(k)}+\left(\boldsymbol{\Phi}_{2}-\boldsymbol{\Phi}_{3} / \tau\right) \boldsymbol{B}_{1}^{(k)}, \\
\boldsymbol{F}_{m-1}=-\boldsymbol{F}_{k+1}, \\
\boldsymbol{F}_{m}=-\boldsymbol{F}_{0, k}, \\
\boldsymbol{\Phi}_{0}=\exp (\boldsymbol{A} \tau), \boldsymbol{\Phi}_{1}=\int_{0}^{\tau} \exp (\boldsymbol{A} \xi) \mathrm{d} \xi, \boldsymbol{\Phi}_{2} \\
=\int_{0}^{\tau} \xi \exp (\boldsymbol{A} \xi) \mathrm{d} \xi, \boldsymbol{\Phi}_{3}=\int_{0}^{\tau} \xi^{2} \exp (\boldsymbol{A} \xi) \mathrm{d} \xi .
\end{gathered}
$$

Eq. (24) can be expressed as the compact form

$$
\boldsymbol{y}_{k+1}=\boldsymbol{E}_{k} \boldsymbol{y}_{k}
$$

where $\boldsymbol{y}_{k}=\operatorname{col}\left(\boldsymbol{x}_{k}, \boldsymbol{x}_{k-1}, \ldots, \boldsymbol{x}_{k-m}\right)$,

$$
\boldsymbol{E}_{k}=\left[\begin{array}{cccccc}
{\left[\boldsymbol{I}-\boldsymbol{F}_{k+1}\right]^{-1}\left(\boldsymbol{F}_{0}+\boldsymbol{F}_{0, k}\right)} & \boldsymbol{0} & \cdots & \boldsymbol{0} & {\left[\boldsymbol{I}-\boldsymbol{F}_{k+1}\right]^{-1} \boldsymbol{F}_{m-1}} & {\left[\boldsymbol{I}-\boldsymbol{F}_{k+1}\right]^{-1} \boldsymbol{F}_{m}} \\
\boldsymbol{I} & \boldsymbol{0} & \cdots & \boldsymbol{0} & \boldsymbol{0} & \boldsymbol{0} \\
\boldsymbol{0} & \boldsymbol{I} & \cdots & \boldsymbol{0} & \boldsymbol{0} & \boldsymbol{0} \\
\vdots & \vdots & \ddots & \vdots & \vdots & \vdots \\
\boldsymbol{0} & \boldsymbol{0} & \cdots & \boldsymbol{I} & \boldsymbol{0} & \boldsymbol{0} \\
\boldsymbol{0} & \mathbf{0} & \cdots & \boldsymbol{0} & \boldsymbol{I} & \boldsymbol{0}
\end{array}\right] .
$$

Using eq. (31), the Floquet matrix can be constructed as

$$
\boldsymbol{\Psi}_{3}=\boldsymbol{E}_{m-1} \boldsymbol{E}_{m-2} \cdots \boldsymbol{E}_{0} .
$$

Based on the Floquet theory, the system stability can be determined by using the spectral radius of $\boldsymbol{\Psi}_{3}$. During the procedure of the FDM, the matrix exponential related functions in eq. (30) all only depend on spindle speed, yet independent of the depth of cut. On the other hand, in eq. (12) of the SDM, the matrix exponential $\mathrm{e}^{\left(\boldsymbol{A}+\boldsymbol{B}_{k}\right) \tau}$ always depends on the spindle speed and depth of cut simultaneously. Hence, the procedure of sweeping the depth of cut and spindle speed plane to determine the stability diagram, the efficiency of the FDM is much higher than that of the SDM. 
The higher order FDM [64] is proposed by employing the higher interpolation schemes for $\boldsymbol{B}(k \tau+\tau-\xi), \boldsymbol{x}(k \tau$ $+\tau-\xi)$ and $\boldsymbol{x}(k \tau+\tau-\xi-T)$. Furthermore, this method can be generalized for the case of systems with multiple time delays [65].

(2) Numerical integration method (NIM). Similar to the TFEA method, for the numerical integration method [62], denote by $t_{0}$ the time the cutter leaves the workpiece, $t_{f}$ the duration of the free vibration after the cutter leaves the workpiece, and $t_{c}$ the duration of the forced vibration. When the cutter leaves the workpiece, $\boldsymbol{B}(\xi)$ in eq. (22)

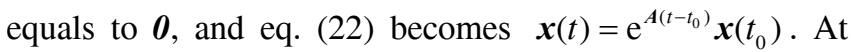
the end of the free vibration, i.e., at $t=t_{0}+t_{f}$, the state item is expressed as

$$
\boldsymbol{x}\left(t_{0}+t_{f}\right)=\exp \left(\boldsymbol{A} t_{f}\right) \boldsymbol{x}\left(t_{0}\right) .
$$

As for the forced vibration duration $\left[t_{0}+t_{f}, t_{0}+T\right]$, divide it equally as $m$ parts, i.e., $\tau=\left(T-t_{f}\right) / m$. Denote by $t_{i}=t_{0}+t_{f}+(i-1) \tau, \quad i=1, \ldots, m+1$ the sampling time points. Note that $\boldsymbol{x}\left(t_{1}\right)=\boldsymbol{x}\left(t_{0}+t_{f}\right)$.

At $t_{i}(i=2, \ldots, m+1), \boldsymbol{x}\left(t_{i}\right)$ can be expressed as

$$
\begin{aligned}
\boldsymbol{x}\left(t_{i}\right)= & \exp \left[A\left(t_{i}-t_{i-1}\right)\right] \boldsymbol{x}\left(t_{i-1}\right)+\int_{t_{i-1}}^{t_{i}}\left\{\exp \left[A\left(t_{i}-\xi\right)\right]\right. \\
& \times \boldsymbol{B}(\xi)[\boldsymbol{x}(\xi)-\boldsymbol{x}(\xi-T)]\} \mathrm{d} \xi
\end{aligned}
$$

which is a Volterra equation of the second kind [66]. Using the trapezium rule [67], eq. (35) can be simplified as

$$
\begin{aligned}
\boldsymbol{x}\left(t_{i}\right)= & \exp \left[\boldsymbol{A}\left(t_{i}-t_{i-1}\right)\right] \boldsymbol{x}\left(t_{i-1}\right) \\
& +\frac{\tau}{2}\left\{\operatorname { e x p } [ \boldsymbol { A } ( t _ { i } - t _ { i - 1 } ) ] \boldsymbol { B } ( t _ { i - 1 } ) \left(\boldsymbol{x}\left(t_{i-1}\right)\right.\right. \\
& \left.\left.-\boldsymbol{x}\left(t_{i-1}-T\right)\right)+\boldsymbol{B}\left(t_{i}\right)\left(\boldsymbol{x}\left(t_{i}\right)-\boldsymbol{x}\left(t_{i}-T\right)\right)\right\} .
\end{aligned}
$$

Combining eqs. (34) and (36), the discrete map is derived as

$$
\left(\boldsymbol{I}-\boldsymbol{C}_{1}-\frac{\tau}{2} \boldsymbol{D}_{1}\right)\left[\begin{array}{c}
\boldsymbol{x}\left(t_{1}\right) \\
\vdots \\
\boldsymbol{x}\left(t_{m+1}\right)
\end{array}\right]=\left(-\frac{\tau}{2} \boldsymbol{D}_{1}+\boldsymbol{E}\right)\left[\begin{array}{c}
\boldsymbol{x}\left(t_{1}-T\right) \\
\vdots \\
\boldsymbol{x}\left(t_{m+1}-T\right)
\end{array}\right],
$$

where

$$
\boldsymbol{C}_{1}=\left[\begin{array}{lllll}
\boldsymbol{0} & & & & \\
\exp (\boldsymbol{A} \tau) & \boldsymbol{0} & & & \\
& \exp (\boldsymbol{A} \tau) & \boldsymbol{0} & & \\
& & \ddots & \ddots & \\
& & & \exp (\boldsymbol{A} \tau) & \boldsymbol{0}
\end{array}\right]
$$

$$
\begin{aligned}
& \boldsymbol{D}_{1}=\left[\begin{array}{lllll}
\boldsymbol{0} & & & & \\
\exp (\boldsymbol{A} \tau) \boldsymbol{B}_{1} & \boldsymbol{B}_{2} & & & \\
& \exp (\boldsymbol{A} \tau) \boldsymbol{B}_{2} & \boldsymbol{B}_{3} & & \\
& & \ddots & \ddots & \\
& & & \exp (\boldsymbol{A} \tau) \boldsymbol{B}_{m} & \boldsymbol{B}_{m+1}
\end{array}\right], \\
& \boldsymbol{E}=\left[\begin{array}{cccc}
\boldsymbol{0} & \cdots & \boldsymbol{0} & \exp \left(\boldsymbol{A} t_{f}\right) \\
\boldsymbol{0} & \cdots & \boldsymbol{0} & \boldsymbol{0} \\
\vdots & \ddots & \vdots & \vdots \\
\boldsymbol{0} & \cdots & \boldsymbol{0} & \boldsymbol{0}
\end{array}\right] \text { and } \boldsymbol{B}_{i}=\boldsymbol{B}\left(t_{i}\right), i=1, \ldots, m+1 .
\end{aligned}
$$

Then, on one tooth passing period, the Floquet matrix $\boldsymbol{\Psi}_{4}$ is obtained

$$
\boldsymbol{\Psi}_{4}=\boldsymbol{J}^{-1} \boldsymbol{L}
$$

where $\boldsymbol{J}=\left(\boldsymbol{I}-\boldsymbol{C}_{1}-\frac{\tau}{2} \boldsymbol{D}_{1}\right), \quad \boldsymbol{L}=\left(-\frac{\tau}{2} \boldsymbol{D}_{1}+\boldsymbol{E}\right)$.

In the same way, the chatter stability can be predicted by using the spectral radius of $\boldsymbol{\Psi}_{4}$ according to the Floquet theory. On this basis, the spectral method with exponential convergence rate [68] and the variable-step integration method suitable for the multiple time delay systems [69] are proposed.

As shown in Table 1, compared eq. (14) via the SDM with eq. (33) via the FDM, and compared eq. (21) via the TFEA method and eq. (38) via the NIM, the integral equation theory provides the milling stability analysis as a general framework.

\section{Applications of the integral equation based algorithms}

Besides chatter stability analysis, FDM and NIM can also be applied for calculation of surface location error (SLE) and sensitivity analysis.

\subsection{Simultaneous prediction of SLE}

As stated in Section 3, the prerequisite of high performance milling is to predict simultaneously chatter stability and dynamic machining error [70]. The FDM in Section 3.3 can be generalized to predict SLE. To discuss the simultaneous prediction problem, eq. (1) should be modified as

Table 1 Comparison of floquet transition matrices

\begin{tabular}{cccc}
\hline \multicolumn{2}{c}{ Time-domain methods } & Floquet matrices & Equations \\
\hline \multicolumn{2}{c}{ SDM [42] } & $\boldsymbol{\Psi}_{1}=\boldsymbol{S}_{m-1} \boldsymbol{S}_{m-2} \cdots \boldsymbol{S}_{0}$ & (14) \\
TFEA [39] & $\boldsymbol{\Psi}_{2}=\boldsymbol{G}^{-1} \boldsymbol{H}$ & $(21)$ \\
Integral & FDM [61] & $\boldsymbol{\Psi}_{3}=\boldsymbol{E}_{m-1} \boldsymbol{E}_{m-2} \cdots \boldsymbol{E}_{0}$ & (33) \\
equation & NIM [62] & $\boldsymbol{\Psi}_{4}=\boldsymbol{J}^{-1} \boldsymbol{L}$ &
\end{tabular}




$$
\boldsymbol{M} \ddot{\boldsymbol{q}}(t)+\boldsymbol{C} \dot{\boldsymbol{q}}(t)+\boldsymbol{K} \boldsymbol{q}(t)=\boldsymbol{K}_{c}(t)[\boldsymbol{q}(t)-\boldsymbol{q}(t-T)]+\boldsymbol{f}_{0}(t),
$$

where

$$
\begin{aligned}
f_{0}(t)= & \sum_{j=1}^{N} a_{p} \cdot g\left(\phi_{j}(t)\right)\left\{f_{t} \cdot\left[\begin{array}{c}
-K_{t} \mathrm{~s} \phi_{j} \mathrm{c} \phi_{j}-K_{n} \mathrm{~s}^{2} \phi_{j} \\
K_{t} \mathrm{~s}^{2} \phi_{j}-K_{n} \mathrm{~s} \phi_{j} \mathrm{c} \phi_{j}
\end{array}\right]\right. \\
& \left.+\left[\begin{array}{c}
-K_{t e} \mathrm{c} \phi_{j}-K_{n e} \mathrm{~s} \phi_{j} \\
K_{t e} \mathrm{~s} \phi_{j}-K_{n e} \mathrm{c} \phi_{j}
\end{array}\right]\right\},
\end{aligned}
$$

is the steady cutting force vector, $\mathrm{s} \phi_{j}=\sin \phi_{j}$ and $\mathrm{c} \phi_{j}=$ $\cos \phi_{j}$.

Eq. (39) can be also rewritten in the state space form

$$
\dot{\boldsymbol{x}}(t)=\boldsymbol{A x}(t)+\boldsymbol{B}(t)[\boldsymbol{x}(t)-\boldsymbol{x}(t-T)]+\boldsymbol{f}(t),
$$

where the matrices $\boldsymbol{A}$ and $\boldsymbol{B}(t)$ are the same as those in eq. (9), $\boldsymbol{f}(t)=\left[\begin{array}{c}0 \\ \boldsymbol{f}_{0}(t)\end{array}\right]$.

Similar to eq. (23), on the interval $[k \tau,(k+1) \tau],(k=$ $0, \cdots, m)$, the solution of eq. (41) can be expressed as $(0 \leqslant t \leqslant \tau)$

$$
\begin{aligned}
\boldsymbol{x}(k \tau+t)= & \exp (\boldsymbol{A} t) \boldsymbol{x}(k \tau)+\int_{0}^{t}\{\exp (\boldsymbol{A} \xi) \boldsymbol{B}(k \tau+t-\xi) \\
& \times[\boldsymbol{x}(k \tau+t-\xi)-\boldsymbol{x}(k \tau+t-\xi-T)]\} \mathrm{d} \xi \\
& +\int_{0}^{t} \exp (\boldsymbol{A} \xi) \boldsymbol{f}(k \tau+t-\xi) \mathrm{d} \xi
\end{aligned}
$$

In eq. (42), letting $t=\tau$, and using linear interpolations for $\boldsymbol{B}(k \tau+\tau-\xi), \quad \boldsymbol{x}(k \tau+\tau-\xi), \quad \boldsymbol{x}(k \tau+\tau-\xi-T)$ and $\boldsymbol{f}(k \tau+\tau-\xi)$, respectively, yielding

$$
\begin{aligned}
\boldsymbol{x}_{k+1}= & {\left[\boldsymbol{I}-\boldsymbol{F}_{k+1}\right]^{-1}\left(\boldsymbol{F}_{0}+\boldsymbol{F}_{0, k}\right) \boldsymbol{x}_{k}+\left[\boldsymbol{I}-\boldsymbol{F}_{k+1}\right]^{-1} \boldsymbol{F}_{m-1} \boldsymbol{x}_{k+1-m} } \\
& +\left[\boldsymbol{I}-\boldsymbol{F}_{k+1}\right]^{-1} \boldsymbol{F}_{m} \boldsymbol{x}_{k-m}+\boldsymbol{P}_{k}
\end{aligned}
$$

where

$$
\boldsymbol{P}_{k}=\boldsymbol{\Phi}_{1} \boldsymbol{f}_{0}^{(k)}+\boldsymbol{\Phi}_{2} \boldsymbol{f}_{1}^{(k)}
$$

Using the vector $\boldsymbol{y}_{k}=\operatorname{col}\left(\boldsymbol{x}_{k}, \boldsymbol{x}_{k-1}, \ldots, \boldsymbol{x}_{k+1-m}, \boldsymbol{x}_{k-m}\right)$, eq. (43) is re-expressed as

$$
\boldsymbol{y}_{k+1}=\boldsymbol{E}_{k} \boldsymbol{y}_{k}+\boldsymbol{Q}_{k} \boldsymbol{P}_{k},
$$

where $\boldsymbol{E}_{k}$ is the same as eq. (32), and

$$
\boldsymbol{Q}_{k}=\operatorname{col}\left(\left[\boldsymbol{I}-\boldsymbol{F}_{k+1}\right]^{-1}, \boldsymbol{0}, \ldots, \boldsymbol{0}\right) .
$$

The state transition relationship on one period can be expressed by the matrices $\boldsymbol{E}_{k}, \boldsymbol{Q}_{k}, \boldsymbol{P}_{k}(k=0, \ldots, m-1)$, i.e.,

$$
\boldsymbol{y}_{m}=\boldsymbol{\Psi}_{3} \boldsymbol{y}_{0}+\boldsymbol{P},
$$

where $\boldsymbol{\Psi}_{3}$ is as shown in eq. (33), and $\boldsymbol{P}$ is defined by

$$
\boldsymbol{P}=\boldsymbol{Q}_{m-1} \boldsymbol{P}_{m-1}+\sum_{i=0}^{m-2}\left(\boldsymbol{E}_{m-1} \boldsymbol{E}_{m-2} \cdots \boldsymbol{E}_{i+1} \boldsymbol{Q}_{i} \boldsymbol{P}_{i}\right)
$$

The steady-state coefficients of the $\ell$-th period is calculated from eq. (47) as

$$
\boldsymbol{y}_{\ell}^{*}=\left(\boldsymbol{I}-\boldsymbol{\Psi}_{3}\right)^{-1} \boldsymbol{P} .
$$

Using $\boldsymbol{y}_{\ell}^{*}$ in eq. (47), the SLE and the vibration induced roughness can be calculated easily [13].

\subsection{Sensitivity analysis of the stability boundary}

Kurdi et al. [72] discussed the sensitivity of the stability boundary by using the TFEA method and finite difference method. Based on the NIM, constructing the Floquet transition matrix, we derive the semi-analytical expressions of the Floquet matrix with respect to machining parameters, without employing the finite difference method [71].

From eq. (38), the derivative of the Floquet matrix $\boldsymbol{\Psi}_{4}$ with respect to the axial depth of cut $a_{p}$ is

$$
\frac{\partial \boldsymbol{\Psi}_{4}}{\partial a_{p}}=\boldsymbol{J}^{-1}\left(-\frac{\tau}{2} \boldsymbol{D}_{1}+\frac{\tau}{2} \boldsymbol{D}_{1} \boldsymbol{J}^{-1} \boldsymbol{L}\right)
$$

The derivative of $\boldsymbol{\Psi}_{4}$ with respect to the spindle speed $\Omega$ is

$$
\frac{\partial \boldsymbol{\Psi}_{4}}{\partial \Omega}=\boldsymbol{J}^{-1}\left(\frac{\partial \boldsymbol{L}}{\partial \Omega}-\frac{\partial \boldsymbol{J}}{\partial \Omega} \boldsymbol{J}^{-1} \boldsymbol{L}\right)
$$

where

$$
\begin{aligned}
& \frac{\partial \boldsymbol{L}}{\partial \Omega}=\left(-\frac{1}{2} \frac{\mathrm{d} \tau}{\mathrm{d} \Omega}\right) \boldsymbol{D}_{1}-\frac{\tau}{2} \cdot \frac{\mathrm{d} \tau}{\mathrm{d} \Omega}\left(\begin{array}{lllllll}
\boldsymbol{0} & & & & & \\
\mathrm{e}^{A \tau} \boldsymbol{B}_{1} & \boldsymbol{0} & & & \\
& \boldsymbol{A e}^{A \tau} \boldsymbol{B}_{2} & \boldsymbol{0} & & \\
& & \ddots & \ddots & \\
& & & \boldsymbol{A e}^{A \tau} \boldsymbol{B}_{m} & \boldsymbol{0}
\end{array}\right) \\
& +\frac{\mathrm{d} t_{f}}{\mathrm{~d} \Omega}\left(\begin{array}{cccc}
\boldsymbol{0} & \cdots & \boldsymbol{0} & \mathrm{e}^{A_{f}} \boldsymbol{A} \\
\boldsymbol{0} & \cdots & \boldsymbol{0} & \boldsymbol{0} \\
\vdots & \ddots & \vdots & \vdots \\
\boldsymbol{0} & \cdots & \boldsymbol{0} & \boldsymbol{0}
\end{array}\right)
\end{aligned}
$$

$$
\begin{aligned}
& \frac{\partial \boldsymbol{J}}{\partial \Omega}=\left(-\frac{1}{2} \frac{\mathrm{d} \tau}{\mathrm{d} \Omega}\right) \boldsymbol{D}_{1}-\frac{\tau}{2} \cdot \frac{\mathrm{d} \tau}{\mathrm{d} \Omega}\left[\begin{array}{llllll}
\boldsymbol{0} & & & & \\
\boldsymbol{A e}^{A \tau} \boldsymbol{B}_{1} & \boldsymbol{0} & & & \\
& \boldsymbol{A e}^{A \tau} \boldsymbol{B}_{2} & \boldsymbol{0} & & \\
& & \ddots & \ddots & \\
& & & \boldsymbol{A \mathrm { e } ^ { A \tau }} \boldsymbol{B}_{m} & \boldsymbol{0}
\end{array}\right] \\
& -\frac{\mathrm{d} \tau}{\mathrm{d} \Omega}\left[\begin{array}{lllll}
0 & & & & \\
A \mathrm{e}^{A \tau} & 0 & & & \\
& A \mathrm{e}^{A \tau} & 0 & & \\
& & \ddots & \ddots & \\
& & & A \mathrm{e}^{A \tau} & 0
\end{array}\right] .
\end{aligned}
$$

Denoting by $\rho$ the ratio of $t_{\mathrm{c}}$ to $T_{\mathrm{l}}$, in eqs. (52), (53), the derivatives of the duration of the free vibration $t_{f}$ and the 
time step $\tau$ with respect to the spindle speed, respectively, are

$$
\begin{gathered}
\frac{\mathrm{d} t_{f}}{\mathrm{~d} \Omega}=-\frac{60}{\Omega^{2} N}(1-\rho), \\
\frac{\mathrm{d} \tau}{\mathrm{d} \Omega}=-\frac{60 \rho}{\Omega^{2} N m} .
\end{gathered}
$$

The derivative of one eigenvalue of the transition matrix $\boldsymbol{\Psi}_{4}$ with respect to one variable $z$ is [73]:

$$
\frac{\partial \lambda}{\partial z}=\frac{\left\langle v, \frac{\partial \boldsymbol{\Psi}_{4}}{\partial z} u\right\rangle}{\langle v, u\rangle}
$$

where $v$ and $u$ are the left and right eigenvectors of the eigenvalue $\lambda$, respectively, and the symbol $\langle\cdot, \cdot\rangle$ represents the inner product of two vectors. The derivative of the most largest module of the eigenvalue with respect to one variable $z$ is

$$
\frac{\partial|\lambda|_{\max }}{\partial z}=\frac{\lambda_{\max } \frac{\bar{\lambda}_{\max }}{\partial z}+\bar{\lambda}_{\max } \frac{\lambda_{\max }}{\partial z}}{2|\lambda|_{\max }}
$$

where the symbol $\bar{\lambda}$ denotes the conjugate of $\lambda$.

At last, similar to [72], for one fixed spindle speed $\Omega_{0}$ and corresponding critical axial depth of cut $a_{\text {lim }}$, the analytical expression of the sensitivity of the stability boundary is obtained

$$
\left.\frac{\mathrm{d} a_{p}}{\mathrm{~d} \Omega}\right|_{\substack{\Omega=\Omega_{0} \\ a_{p}=a_{\text {lim }}}}=-\left.\frac{\partial|\lambda|_{\max }}{\partial \Omega}\right|_{\substack{\Omega=\Omega_{0} \\ a_{p}=a_{\mathrm{lim}}}} /\left.\frac{\partial|\lambda|_{\max }}{\partial a_{p}}\right|_{\substack{\Omega=\Omega_{p} \\ a_{p}=a_{\mathrm{lim}}}} .
$$

Eq. (58) serves as a basis for gradient-based optimization of machining parameters and uncertainty analysis of the machining process.

\section{Conclusion and future work}

The paper review the time-domain semi-analytical methods for milling process stability analysis, and states the similarities and differences of the SDM, TFEA method, FDM and NIM, with emphasis on the FDM and NIM in the framework of integral equations.

To break the conventional idea constructing the approximate algorithms in the differential equation framework, the FDM describes the dynamics of milling processes in the framework of integral equations. Based on this integral equation, the time-delay item, the state item, and the periodic coefficients are simultaneously linearly interpolated. The Floquet transition matrix is then semi-analytically constructed on a single period. The matrix exponential related functions involved in the calculation procedure depend only on the spindle speed, independent of the depth of cut. Therefore the FDM has a high computational efficiency. This method has four advantages: (1) suitable for large/ small radial depth of cut, large/small axial depth of cut, thin-wall milling and other operations; (2) simultaneous prediction of the surface position error (SLE); (3) it can be applied to the cases of considering the nonlinear cutting force models, varying-speed, etc.; (4) it can be applied to the multiple time delay milling systems, providing a basis for simultaneous optimization of cutter parameters and machining process parameters. On the basis of the FDM, the numerical integration method is developed by using the classical techniques of integral equations. Then, the derivatives of the Floquet transition matrix with respect to the processing parameters are obtained, which lays the foundation for the gradient-based optimization of the milling process parameters.

The further development of the milling process dynamics and stability analysis method should focus on the following aspects. (1) We should build up more accurate dynamic models of 5-axis milling processes, such as for flank milling dynamic model of the distribution parameters, models for the difficult-to-cut materials, low-speed cutting process damping models and a more comprehensive machine-toolworkpiece-fixture system dynamic model. (2) We should propose the active control strategy based on the dynamic model. To break through the conventional strategy selecting the machining parameters in the plane of the spindle speed and depth of cut select to avoid chatter vibrations, we should design the active control system, develop devices and sensors to monitor and control the machining processes, and enlarge the stable area of the system. (3) We should integrate the physical model of the machining process with the dynamic model. To break through the limitations of conventional milling dynamic model for prediction of the geometric accuracy of machined surfaces, we should fuse the process dynamics with the multi-physics model [74-77], developing efficient numerical methods to explore the macro/micro performance of machined surfaces with respect to process parameters. (4) The last one is to fuse the dynamics model with some advanced machine tool control strategies [78-88] for high performance control ensuring high performance machining.

This work was supported by the National Basic Research Program of China (2011CB706804) and the National Natural Science Foundation of China (51120155001, 51121002).

1 Zhu L M, Ding H, Xiong Y L. Third-order point contact approach for five-axis sculptured surface machining using non-ball-end tools (I): Third-order approximation of tool envelope surface. Sci China Technol Sci, 2010, 53: 1904-1912

2 Zhu L M, Ding H, Xiong Y L. Third-order point contact approach for five-axis sculptured surface machining using non-ball-end tools (II): Tool positioning strategy. Sci China Technol Sci, 2010, 53: 21902197 
3 Ye T, Xiong C H, Xiong Y L, et al. Kinematics constrained five-axis tool path planning for high material removal rate. Sci China Technol Sci, 2011, 54: 3155-3165

4 Guo Q, Sun Y W, Guo D M. Analytical modeling of geometric errors induced by cutter runout and tool path optimization for five-axis flank machining. Sci China Technol Sci, 2011, 54: 3180-3190

5 Bi Q Z, Wang Y H, Zhu L M, et al. Wholly smoothing cutter orientations for five-axis NC machining based on cutter contact point mesh. Sci China Technol Sci, 2010, 53: 1294-1303

6 Wiercigroch M, Budak E. Sources of nonlinearities, chatter generation and suppression in metal cutting. Phil Trans R Soc A, 2001, 359: 663-693

7 Ismail F, Vadari V R. Machining chatter of end mills with unequal modes. J Eng Ind-T ASME, 1990, 112: 229-235

8 Wiercigroch M, Krivtsov A M. Frictional chatter in orthogonal metal cutting. Phil Trans R Soc A, 2001, 359: 713-738

9 Davies M A, Burns T J. Thermomechanical oscillations in material flow during high-speed machining. Phil Trans R Soc A, 2001, 359: $821-846$

10 Altintas Y, Weck M. Chatter stability of metal cutting and grinding. CIRP Ann-Manuf Techn, 2004, 53: 619-642

11 Altintas Y, Stépán G, Merdol D, et al. Chatter stability of milling in frequency and discrete time domain. CIRP J Manuf Sci Techn, 2008, 1: $35-44$

12 Schmitz T, Ziegert J. Examination of surface location error due to phasing of cutter vibrations. Precis Eng, 1999, 23: 51-62

13 Bachrathy D, Insperger T, Stepan G. Surface properties of the machined workpiece for helical mills. Mach Sci Technol, 2009, 13: 227-245

14 Altintas Y. Manufacturing Automation: Metal Cutting Mechanics, Machine Tool Vibrations, and CNC Design. Cambridge: Cambridge University Press, 2000

15 Gradisek J, Kalveram M, Insperger T, et al. On stability prediction for milling. Int J Mach Tool Manu, 2005, 45: 769-781

16 Mann B P, Young K A, Schmitz T L, et al. Simultaneous stability and surface location error predictions in milling. J Manuf Sci E-T ASME, 2005, 127: 446-453

17 Schmitz T L. Predicting high-speed machining dynamics by substructure analysis. CIRP Ann-Manuf Techn, 2000, 49: 303-308

18 Schmitz T L, Davies M A, Kennedy M D. Tool point frequency response prediction for high-speed machining by RCSA. J Manuf Sci E-T ASME, 2001, 123: 700-707

19 Zhu R X, Kapoor S G, Devor R E. Mechanistic modeling of the ball end milling process for multi-axis machining of free-form surfaces. $\mathrm{J}$ Manuf Sci E-T ASME, 2001, 123: 369-379

20 Budak E, Altintas Y, Armarego E J A. Prediction of milling force coefficients from orthogonal cutting data. J Manuf Sci E-T ASME, 1996, 118: 216-224

21 Budak E, Ozturk E, Tunc L T. Modeling and simulation of 5-axis milling processes. CIRP Ann-Manuf Techn, 2009, 58: 347-350

22 Zhang X J, Xiong C H, Ding Y. A new solution for stability prediction in flexible part milling. Lect Notes Comput Sci, 2011, 7102: $452-464$

23 Zhang X J, Xiong C H, Ding Y, et al. Milling stability analysis with simultaneously considering the structural mode coupling effect and regenerative effect. Int J Mach Tool Manu, 2011, 53: 127-140

24 Mao X Y, Liu H Q, Li B. Time-frequency analysis and detecting method research on milling force token signal in spindle current signal. Sci China Ser E-Technol Sci, 2009, 52: 2810-2813

25 Liu H Q, Chen Q H, Li B, et al. On-line chatter detection using servo motor current signal in turning. Sci China Technol Sci, 2011, 54: 3119-3129

26 Sridhar R, Hohn R E, Long G W. A stability algorithm for the general milling process. J Eng Ind-T ASME, 1968, 90: 330-334

27 Tlusty J, Ismail F. Basic non-linearity in machining chatter. CIRP Ann-Manuf Techn, 1981, 30: 299-304

28 Tlusty J, Ismail F. Special aspects of chatter in milling. J Vib Acoust Stress Reliab Des-T ASME, 1983, 105: 24-32

29 Smith S, Tlusty J. Efficient simulation programs for chatter in milling.
CIRP Ann-Manuf Techn, 1993, 42: 463-466

30 Davies M A, Pratt J R, Dutterer B S, et al. Stability of low radial immersion milling. CIRP Ann-Manuf Techn, 2000, 49: 37-40

31 Davies M A, Pratt J R, Dutterer B, et al. Stability prediction for low radial immersion milling. J Manuf Sci E-T ASME, 2002, 124: 217225

32 Campomanes M L, Altintas Y. An improved time domain simulation for dynamic milling at small radial immersions. J Manuf Sci E-T ASME, 2003, 125: 416-422

33 Li Z Q, Liu Q. Solution and analysis of chatter stability for end milling in the time-domain. Chin J Aeronaut, 2008, 21: 169-178

34 Minis I, Yanushevsky R. A new theoretical approach for the prediction of machine tool chatter in milling. J Eng Ind-T ASME, 1993, 115: $1-8$

35 Altintas Y, Budak E. Analytical prediction of stability lobes in milling. CIRP Ann-Manuf Techn, 1995, 44: 357-362

36 Budak E, Altintas Y. Analytical prediction of chatter stability in milling-part I: General formulation. J Dyn Syst-T ASME, 1998, 120: 22-30

37 Merdol S D, Altintas Y. Multi frequency solution of chatter stability for low immersion milling. J Manuf Sci E-T ASME, 2004, 126: 459466

38 Bayly P V, Halley J E, Mann B P, et al. Stability of interrupted cutting by temporal finite element analysis. Proc ASME Des Eng Tech Conf, 2001, 6C: 2361-2370

39 Mann B P, Patel B R. Stability of delay equations written as state space models. J Vib Control, 2010, 16: 1067-1085

40 Garg N K, Mann B P, Kim N H, et al. Stability of a time-delayed system with parametric excitation. J Dyn Syst-T ASME, 2007, 129: 125-135

41 Insperger T, Stépán G. Semi-discretization method for delayed systems. Int J Number Meth Eng, 2002, 55: 503-518

42 Insperger T, Stépán G. Updated semi-discretization method for periodic delay-differential equations with discrete delay. Int J Number Meth Eng, 2004, 61: 117-141

43 Insperger T, Stépán G, Turi J. On the higher-order semi-discretizations for periodic delayed systems. J Sound Vib, 2008, 313: 334-341

44 Long X H, Balachandran B, Mann B P. Dynamics of milling processes with variable time delays. Nonlinear Dynam, 2007, 47: 49-63

45 Long X H, Balachandran B. Stability of up-milling and down-milling operations with variable spindle speed. J Vib Control, 2010, 16: 1151-1168

46 Li Z W, Long X H, Meng G. Stability analysis for milling process by semidiscretization method based on Magnus-Guassian truncation. J Vib Shock, 2009, 28: 69-73

47 Song Q H, Ai X, Wan Y, et al. Stability of milling system with variable radial depth of cut considering runout effect. J Vib Meas Diagn, 2008, 28: 206-210

48 Song Q H, Ai X, Wan Y, et al. Stability prediction for high-speed milling including feed rate in low radial immersion (in Chinese). China Mech Eng, 2008, 19: 1148-1152

49 Wan M, Zhang W H, Dang J W, et al. A unified stability prediction method for milling process with multiple delays. Int J Mach Tool Manu, 2010, 50: 29-41

50 Olgac N, Hosek M. A new perspective and analysis for regenerative machine tool chatter. Int J Mach Tool Manu, 1998, 38: 783-798

51 Olgac N, Sipahi R. Dynamics and stability of variable-pitch milling. J Vib Control, 2007, 13: 1031-1043

52 Maghami Asl F, Ulsoy A G. Analysis of a system of linear delay differential equations. J Dyn Syst-T ASME, 2003, 125: 215-223

53 Yi S, Nelson P W, Ulsoy A G. Delay differential equations via the matrix Lambert W function and bifurcation analysis: Application to machine tool chatter. Math Biosci Eng, 2007, 4: 355-368

54 Butcher E A, Ma H, Bueler E, et al. Stability of linear time-periodic delay-differential equations via Chebyshev polynomials. Int J Number Meth Eng, 2004, 59: 895-922

55 Butcher E A, Bobrenkov O A, Bueler E, et al. Analysis of milling stability by the Chebyshev collocation method: Algorithm and optimal stable immersion levels. J Comput Nonlin Dyn, 2009, 4: 031003 
56 Schmitz T L, Mann B P. Closed-form solutions for surface location error in milling. Int J Mach Tool Manu, 2006, 46: 1369-1377

57 Mann B P, Bartow M J, Young K A, et al. Machining accuracy due to tool or workpiece vibrations. ASME, Manuf Eng Div, MED, 2003, 14: 55-62

58 Mann B P, Edes B T, Easley S J, et al. Chatter vibration and surface location error prediction for helical end mills. Int J Mach Tool Manu, 2008, 48: 350-361

59 Insperger T, Gradisek J, Kalveram M, et al. Machine tool chatter and surface location error in milling processes. J Manuf Sci E-T ASME, 2006, 128: 913-920

60 Bayly P V, Mann B P, Schmitz T L, et al. Effects of radial immersion and cutting direction on chatter instability in end-milling. ASME, Manuf Eng Div, MED, 2002, 13: 351-363

61 Ding Y, Zhu L M, Zhang X J, et al. A full-discretization method for prediction of milling stability. Int J Mach Tool Manu, 2010, 50: 502509

62 Ding Y, Zhu L M, Zhang X J, et al. Numerical integration method for prediction of milling stability. J Manuf Sci E-T ASME, 2011, 133: 031005

63 Bayly P V, Halley J E, Mann B P, et al. Stability of interrupted cutting by temporal finite element analysis. J Manuf Sci E-T ASME, 2003, 125: 220-225

64 Ding Y, Zhu L M, Zhang X J, et al. Second-order full-discretization method for milling stability prediction. Int J Mach Tool Manu, 2010, 50: 926-932

65 Zhang X J, Xiong C H, Ding Y. Improved full-discretization method for milling chatter stability prediction with multiple delays. Lect Notes Comput Sci, 2010, 6425: 541-552

66 Delves L M, Mohamed J L. Computational Methods for Integral Equations. Cambridge: Cambridge University Press, 1985

67 Yang W Y, Cao W, Chung T S, et al. Applied numerical methods using Matlab. Hoboken N. J.: Wiley-Interscience, 2005

68 Ding Y, Zhu L M, Zhang X J, et al. Milling stability analysis using the spectral method. Sci China Technol Sci, 2011, 54: 3130-3136

69 Zhang X J, Xiong C H, Ding Y, et al. Variable-step integration method for milling chatter stability prediction with multiple delays. Sci China Technol Sci, 2011, 54: 3137-3154

70 Ding Y, Zhu L M, Zhang X J, et al. On a numerical method for simultaneous prediction of stability and surface location error in low radial immersion milling. J Dyn Syst-T ASME, 2011, 133: 024503

71 Ding Y, Zhu L M, Zhang X J, et al. Response sensitivity analysis of the dynamic milling process based on the numerical integration method. Chin J Mech Eng, 2012, 25: 940-946

72 Kurdi M H, Haftka R T, Schmitz T L, et al. A robust semi-analytical method for calculating the response sensitivity of a time delay system. $\mathrm{J}$ Vib Acoust-T ASME, 2008, 130: 064504

73 Lax P D. Linear Algebra and Its Applications. New York: Wiley-
Interscience, 2007

74 Ji C H, Liu Z Q, Liu L N. Simulation and analysis of aerodynamics for high speed face milling cutters. Sci China Technol Sci, 2010, 53: 2575-2580

75 Guo Y B, Liang Y C, Chen M J, et al. Molecular dynamics simulations of thermal effects in nanometric cutting process. Sci China Technol Sci, 2010, 53: 870-874

76 Chen M J, Xiao G B, Chen J X, et al. Research on the influence of machining introduced sub-surface defects and residue stress upon the mechanical properties of single crystal copper. Sci China Technol Sci, 2010, 53: 3161-3167

77 Zhang S Q, Guo W L, Li H, et al. Experimental investigation of three-dimensional mixed-mode fracture of a titanium alloy at room and elevated temperatures. Sci China Technol Sci, 2011, 54: 27602767

78 Zhang L X, Sun R Y, Gao X S, et al. High speed interpolation for micro-line trajectory and adaptive real-time look-ahead scheme in CNC machining. Sci China Technol Sci, 2011, 54: 1481-1495

79 Fan S X, Nagamune R, Fan D P. Double-loop robust tracking control for micro machine tools. Sci China Technol Sci, 2011, 54: 30543063

80 Zhou C, Yang H Y, Yang L K, et al. Piecewise model and experiment of power chuck's gripping force loss during high speed turning. Sci China Technol Sci, 2011, 54: 972-982

81 Sun T, Song Y M, Li Y G, et al. Dimensional synthesis of a 3-DOF parallel manipulator based on dimensionally homogeneous Jacobian matrix. Sci China Technol Sci, 2010, 53: 168-174

82 Yu J J, Dai J S, Bi S S, et al. Type synthesis of a class of spatial lower-mobility parallel mechanism with orthogonal arrangement based on lie group enumeration. Sci China Technol Sci, 2010, 53: 388-404

83 Zhao C Y, Wen B C, Zhang X L. Synchronization of the four identical unbalanced rotors in a vibrating system of plane motion. Sci China Technol Sci, 2010, 53: 405-422

84 Zhang Y T, Mu D J. New concept and new theory of mobility calculation for multi-loop mechanisms. Sci China Technol Sci, 2010, 53: 1598-1604

85 Li R Q, Xiao D H, Dai J S. Orientation angle rotatability of planar serial n-link manipulators. Sci China Technol Sci, 2010, 53: 1620-1637

86 Liu X J, Li Z D, Chen X. A new solution for topology optimization problems with multiple loads: The guide-weight method. Sci China Technol Sci, 2011, 54: 1505-1514

87 Zhang Y T, Li Y W, Wang L Y. A new formula of mechanism mobility based on virtual constraint loop. Sci China Technol Sci, 2011, 54: 2768-2775

88 Zeng D X, Huang Z. Type synthesis of the rotational decoupled parallel mechanism based on screw theory. Sci China Technol Sci, 2011, 54: $998-1004$

Open Access This article is distributed under the terms of the Creative Commons Attribution License which permits any use, distribution, and reproduction in any medium, provided the original author(s) and source are credited. 